EPJ Web of Conferences 52, 07001 (2013)

DOI: $10.1051 /$ epjconf/20135207001

C) Owned by the authors, published by EDP Sciences, 2013

\title{
Interactions of Cosmic Rays in the primary energy range (0.1-1) PeV studied by the ARGO-YBJ Experiment
}

\author{
A.D'Amone ${ }^{1,2}$, I.De Mitri ${ }^{1,2}$, and A.Surdo, a (on behalf of ARGO-YBJ Collaboration) \\ ${ }^{1}$ Dipartimento di Matematica e Fisica, "E. De Giorgi", Università del Salento, via per Arnesano, 73100, Lecce, Italy \\ ${ }^{2}$ Istituto Nazionale di Fisica Nucleare (INFN), Sezione di Lecce, via per Arnesano, 73100, Lecce, Italy
}

\begin{abstract}
The ARGO-YBJ detector layout, features and location offer a unique possibility for a detailed study of several characteristics of the hadronic component of the cosmic ray flux in an energy range hardly accessed by direct measurements. The proton-air cross section has already been measured by ARGO-YBJ in an energy region up to $\sim 100 \mathrm{TeV}$ where the primary cosmic ray composition is sufficiently well known for that measure to be unbiased. The analog readout of the RPC signals now provides new tools to measure the lateral distribution of particle density (LDF) as close as a few meters from the core and to investigate with unprecedented resolution the shower time structure. This allows to extend the hadronic interaction and mass composition studies up to $\sim \mathrm{PeV}$ energies in the laboratory rest frame. Moreover, it is shown that the LDF of detected showers can be properly described, even very close to the shower axis, by a NKG-like function, whose shape parameter is clearly related to the shower age and can be used for studying the mass composition of cosmic rays.
\end{abstract}

\section{Introduction}

As known, cosmic rays (CR) can be measured only indirectly above $\mathrm{E} \sim 10^{14} \mathrm{eV}$, through the cascades of secondary particles (EAS) produced by their interactions in the atmosphere and detected by surface apparatuses, at sea level or at mountain altitudes. The primary particle parameters are then inferred by simulating the generation of EAS through Monte Carlo (MC) and comparing predictions with measurements, so the modeling of hadronic multiparticle production in air-shower simulations becomes crucial. Indeed, while the electroweak interaction part is well described, the hadronic interaction processes are relatively poorly known or subject to large theoretical uncertainties. As a consequence, the interpretation of experimental air shower data is highly affected by the uncertainty of the hadronic interaction generators in MC simulations.

The Large Hadron Collider (LHC) at CERN allows for the first time to access the energy region above the knee of the primary CR stectrum $(\sim 3 \mathrm{PeV})$, so the analysis of LHC inclusive particle data is particularly interesting for constraining existing hadronic interaction models and for testing possible new mechanisms of hadron production. Nevertheless, CR experiments can also play an important role in studying the hadronic interaction features, since they can explore energy ranges not covered by accelerators and investigate kinematic regions hardly accessed by collider experiments.

In this context, the ARGO-YBJ experiment, with its detector features and location, can offer a unique chance for a detailed study of several characteristics of

\footnotetext{
a e-mail: surdo@le.infn.it
}

the hadronic component of the primary CRs, in an energy range beyond the direct measurement current limits.

A fundamental quantity of all CR interaction models is the total hadronic cross section $\sigma_{\text {tot }}$ and its elastic and inelastic (in particular, diffractive) components. Previous ARGO-YBJ results on hadronic interactions concern the measurement of the proton-air cross section in the energy range 1-100 $\mathrm{TeV}$ and, consequently, of the total p$\mathrm{p}$ cross section at center of mass energies $\sqrt{s}$ between 70 and $500 \mathrm{GeV}$ [1]. The ARGO-YBJ result is consistent with the general trend of experimental data, favouring an asymptotic $\ln ^{2}(s)$ rise of the cross section. The analysis, based on the flux attenuation at different atmospheric depths (i.e. zenith angles), used the digital information only (see next section) and exploited the detector capabilities of constraining the shower development stage by means of hit multiplicity and spatial concentration.

The analysis presented here is based on the data taken with the RPC analog charge readout that in fact extends the investigable $\mathrm{CR}$ energy range and allows to study primary collisions with $\sqrt{s}$ in the TeV region. Further improvements in the analysis come from the use of the detailed information on the shower front that ARGO-YBJ is able to record with very high precision. A preliminary analysis using a sub-set of these data has been presented in [2].

\section{The ARGO-YBJ experiment}

The ARGO-YBJ detector is a full coverage extensive air shower array made by a single layer of Resistive Plate Chambers (RPCs) operated in streamer mode. The array, 


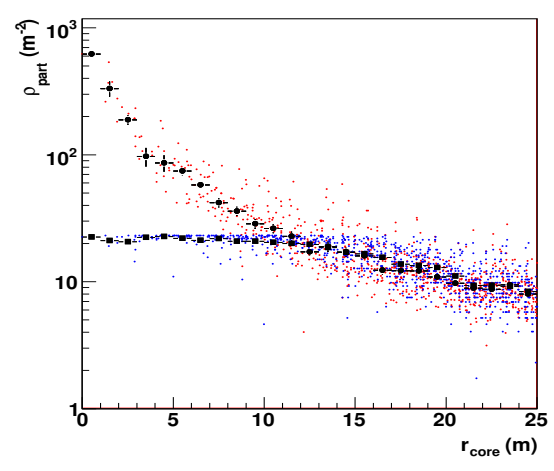

Figure 1. Particle lateral distribution of a real event detected by ARGO-YBJ. Strips (blue dots) are saturated up to $\sim 15 \mathrm{~m}$ from the core, while the particle density measured with the analog RPC charge (red dots) exhibits no saturation also very near to the core.

located in the YanBaJing (Tibet, China) Cosmic Ray Laboratory, at an altitude of $4300 \mathrm{~m}$ above sea level (corresponding to a vertical atmospheric depth of $\sim 606 \mathrm{~g} / \mathrm{cm}^{2}$ ), was running since November 2007 until February 2013. It was organized in 153 clusters of 12 RPCs. Each RPC was read out by ten $62 \times 56 \mathrm{~cm}^{2}$ pads, which were further divided into 8 strips, thus providing a larger particle counting dynamic range $[3,4]$. The signals coming from all the strips of a given pad were sent to the same channel of a multihit TDC. The whole system provided a single hit (pad) time resolution of $1.8 \mathrm{~ns}$, which, together with the size of space pixels (strips) and the full-coverage layout, allowed an event imaging and reconstruction with very high details. A system for the RPC analog charge readout [5] from larger pads (BigPads), $124 \times 140 \mathrm{~cm}^{2}$, each one covering half a chamber, was implemented and took data since January 2010. This actually extended the detector operating range from about $100 \mathrm{TeV}$ up to $\mathrm{PeV}$ primary energies. Moreover, the RPC charge information allows measuring very high particle densities, without the saturation at $\sim 23 / \mathrm{m}^{2}$ otherwise present by using the detector in digital mode, i.e. by counting the fired strips (see Fig. 1).

The system used a set of dynamic gain scales, with an overlap between digital and analog linearity ranges, thus providing an efficient way to calibrate the system itself $[6,7]$. The different scales of operation also define the ranges of the maximum number of particles measured by the BigPads, as shown in Fig. 2 which reports the differential rate of the maximum for three of that scales. Noticeably, there is a good overlap for this quantity as measured at the different scales; moreover, no saturation of the detector response is evident in the plot behaviour; finally, the measurable maximum density of particles covers more than two decades, while the event rate spans over five decades. Apart the data of the first scale, useful for calibration purposes, for this analysis we used two sets of analog data collected with the second and third gain scale, thus accessing particle density values up to $\sim 10^{4} / \mathrm{m}^{2}$.

The peculiar features of the detector, that is fullcoverage and charge readout segmentation, joined to the dynamical operating range of the charge readout system

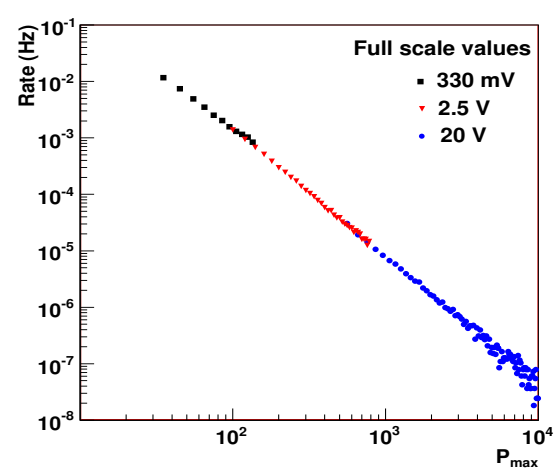

Figure 2. Differential rate of particle maximum on a BigPad for events with the core inside the central carpet and $\theta \leq 15^{\circ}$. The leftmost (most sensitive) scale widely overlaps the digital range.

allow to study the detailed structure of the particle density distribution for $\sim 30 \mathrm{~m}$ starting from the core at ground. On the basis of such information, several characteristics of the hadronic interactions could be investigated and different hadronization models compared to check their validity. In particular, the distribution and physical properties of secondary particles produced in the very forward kinematic region of the primary interaction can be in principle inspected. Even if the lateral distribution of particles at ground is rather determined by multiple Coulomb scattering and by the $\mathrm{p}_{T}$ spectra of secondaries at much lower energies, such a measurement is important for checking the overall physics consistency of soft and hard interaction mechanisms implemented in the models.

\section{Analysis strategy and objectives}

The detailed study of the lateral particle density profile (LDF) at ground is expected to provide information on the longitudinal profile of the showers in the atmosphere, that is to estimate their development stage, or 'age', which essentially is expressed by $X_{\max }\left(\mathrm{g} / \mathrm{cm}^{2}\right)$, the atmospheric depth at which the cascade reaches its maximum size. If true, this implies the possibility of selecting some intervals of $X_{\max }$ or, equivalently, of $X_{d m}$, the distance between the shower maximum and the detector. The selection of showers with $X_{d m}$ in a given interval for different zenith angles is an important point for the measurement of $\mathrm{p}$-air cross section using the method of flux attenuation.

The shower development stage in the atmosphere, as observed at a fixed altitude (the detection one), depends on the energy of the interacting primary. For fixed energy, it depends on the primary nature: heavier primaries interact higher in the atmosphere, thus giving showers which, on average, reach their maximum at a greater distance from the detector than a lighter primary of the same energy. For this reason, if the shower (i.e. primary) energy can be determined independently, the measure of its development stage, that is its 'longitudinal age', is expected to be sensible to the primary nature. An EAS array by itself cannot measure directly the shower development stage, 


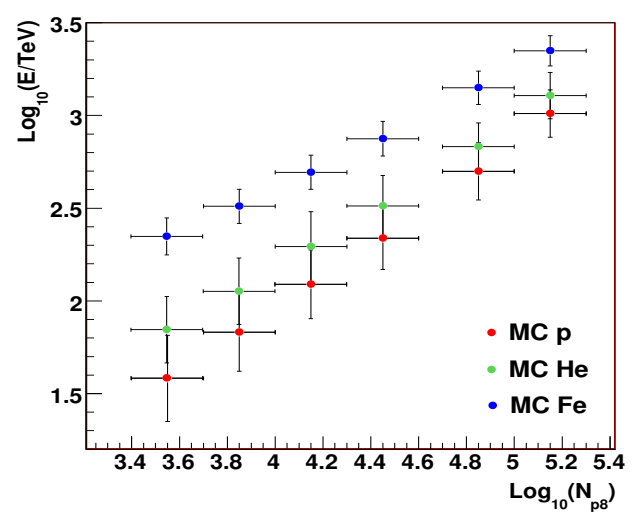

Figure 3. Primary energy for $\mathrm{p}, \mathrm{He}$ and $\mathrm{Fe}$ initiated $\mathrm{MC}$ showers vs the number of particles detected at ground within $8 \mathrm{~m}$ from the shower core. Vertical error bars are the RMSs of the energy distributions, while horizontal ones refer to the adopted $N_{p 8}$ bins.

through the determination of the depth of the shower maximum. It can only measure the particle density distribution at ground as a function of the core distance (LDF) and from the slope of this distribution get information on the longitudinal shower development. Historically, it was shown that the LDF (at least of electrons), as measured by a traditional sampling EAS array at distances of the order of hundred meters from the core, can be described by the Nishimura-Kamata-Greisen (NKG) structure function [8], with parameters reflecting the shower size, the detection altitude and the shower age. The age parameter determined in this way is usually referred to as 'lateral age', since obtained from the LDF [9]. It, in principle, coincides with the 'longitudinal age' both for purely e.m. and hadron initiated EAS, but, in an experimental context, they can differ, since most showers come from hadrons and the two quantities are measured with completely different techniques. However, they must be strongly related.

Other important points for the analysis are then: an estimator of the energy to be assigned to the primary originating the detected shower (at least for well reconstructed events), the capability to reject as many as possible showers induced by heavy primary nuclei (starting from $\alpha$ particles), using the event characteristics and observables provided by the detector. This would give the possibility of selecting a proton-enriched event sample, thus permitting a reliable measurement of the p-air cross-section. A possible residual contamination will contribute to the overall systematic uncertainty of the measure. For these purposes, a full MC simulation has been employed, including the shower production and transport in the atmosphere, the ARGO-YBJ detector response and the event reconstruction with the same code as for real data.

\section{Monte Carlo simulation}

Several samples of simulated data were produced and studied with the aim of correlating the experimental observables to the physics parameters of the showers. About $30 \cdot 10^{6}$ proton, $7 \cdot 10^{6} \alpha$ particle and $3.5 \cdot 10^{6}$ iron nucleusinitiated showers were generated by the CORSIKA [10]

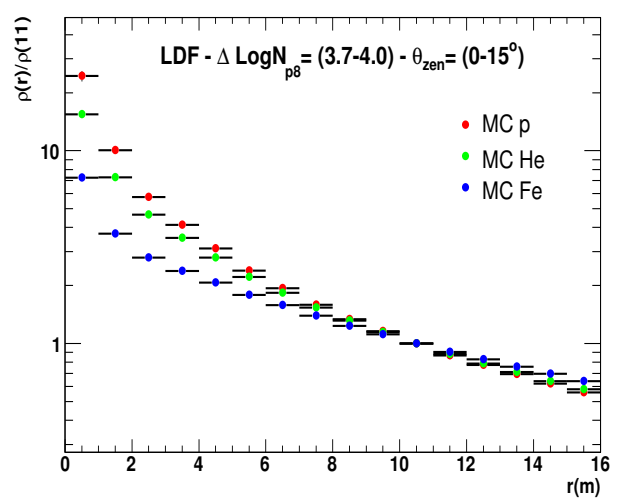

Figure 4. Reconstructed LDF of the detected particles around the core position at ground for simulated $\mathrm{p}, \mathrm{He}$ and $\mathrm{Fe}$ initiated showers with $10^{3.7}<N_{p 8}<10^{4.0}$. The three distribution are normalized to the particle density at $11 \mathrm{~m}$ of core distance.

code, with zenith angle up to $30^{\circ}$, energy range between $1 \mathrm{TeV}$ and $3000 \mathrm{TeV}$ and spectral index as given in the review [12]. In order to have a better evaluation of systematics, we produced independent samples of proton showers by using two different hadronic interaction models, namely QGSJET-II.03 [13] and SIBYLL-2.1 [14]. If not differently stated, the results described in this work refer to QGSJET-II model. The full simulation of the detector response, based on the GEANT package [11], included the effects of time resolution, trigger logic, electronics noise, etc. Simulated data have been analyzed by using the same reconstruction code as for real data. The simulation reliability was successfully checked by comparing several simulated and measured quantities.

Events that triggered the analog RPC readout $(\geq 73$ fired pads in a cluster) were subsequently selected by requiring the core position, reconstructed with a precision of the order of $\sim 1 \mathrm{~m}$ or less, in a fiducial area $64 \times 64 \mathrm{~m}^{2}$ around the detector center. This cut actually reduces to a negligible value $\left(<10^{-3}\right)$ the fraction of events with true core outside the detector but mis-reconstructed inside. This preliminary work was also restricted to events with reconstructed zenith angle $\theta<15^{\circ}$.

As a first step, various observables were considered and analyzed in order to find a suitable estimator of the primary CR energy $E$. Among them, $N_{p 8}$, the number of particles detected at ground within a distance of $8 \mathrm{~m}$ from the shower core at ground, resulted well correlated with $E$, not biased by the finite detector size and only slightly affected by shower to shower fluctuations. In Fig. 3, the correlation between $E$ and $N_{p 8}$ is shown for simulated proton, helium and iron-nucleus initiated showers. So, five $N_{p 8}$ intervals have been chosen to select event samples corresponding to different primary energies.

Several quantities, such as the LDF of the detected particles around the core, estimated through the analog system simulation, have been checked to investigate the systematics introduced by the used hadronic interaction model. The reconstructed LDF obtained with QGSJET-II and SIBYLL-2.1 are quite similar, their difference being within few percent, as expected in this energy range [2]. 
Table 1. Summary of $s^{\prime}$ values from LDF fit by the function (1) for MC p, He, Fe-initiated shower samples and experimental data. The errors are only statistical (given by the fits) and do not include the systematic uncertainties due to intrinsic shower fluctuations.

\begin{tabular}{cccccc}
\hline$\Delta \log \left(N p_{8}\right)$ & $\begin{array}{c}\langle\text { Energy }\rangle(\mathrm{TeV}) \\
\mathrm{p} / \mathrm{He} / \mathrm{Fe}\end{array}$ & $\mathrm{p}-s^{\prime}$ & $\mathrm{He}-s^{\prime}$ & $\mathrm{Fe}-s^{\prime}$ & DATA- $s^{\prime}$ \\
& $40 / 70 / 200$ & $1.31 \pm 0.01$ & $1.47 \pm 0.01$ & $1.73 \pm 0.01$ & $1.412 \pm 0.002$ \\
$3.4-3.7$ & $70 / 100 / 300$ & $1.28 \pm 0.01$ & $1.40 \pm 0.01$ & $1.66 \pm 0.01$ & $1.400 \pm 0.003$ \\
$3.7-4.0$ & $200 / 300 / 750$ & $1.20 \pm 0.01$ & $1.29 \pm 0.01$ & $1.55 \pm 0.01$ & $1.391 \pm 0.006$ \\
$4.3-4.6$ & $500 / 700 / 1400$ & $1.17 \pm 0.01$ & $1.26 \pm 0.01$ & $1.47 \pm 0.01$ & $1.33 \pm 0.01$ \\
$4.7-5.0$ & $1.00 / 1300 / 2200$ & $1.12 \pm 0.02$ & $1.25 \pm 0.02$ & $1.43 \pm 0.02$ & $1.32 \pm 0.03$ \\
$5.0-5.3$ & $1000 /$ \\
\hline
\end{tabular}

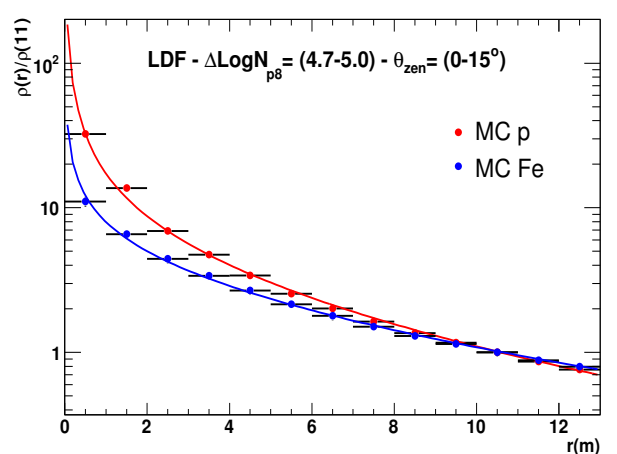

Figure 5. Fits to the reconstructed LDFs of the detected particles around the axis for simulated proton and iron-nucleus induced showers with $10^{4.7}<N_{p 8}<10^{5.0}$, by the NKG-like function (1).

As already stated in the previous section, the basic idea is to get information on the shower development stage from the LDF structure around the core. We outline that no other experiment is able to measure the LDF at small distance (a few meters) from the core with great accuracy and try to correlate it with the shower development characteristics, as ARGO-YBJ in reverse is capable to do. Firstly, we found a promising correlation between the reconstructed LDF slope in the range $1 \div 2 \mathrm{~m}$ from the core and the distance of the shower maximum from the detection level, $X_{d m}$, for $\mathrm{p}$ and iron initiated showers, as reported in [2]. Then, the whole reconstructed LDFs (up to $10-15 \mathrm{~m}$ of distance from the core), for different $N_{p 8}$ intervals and different shower initiating primaries, have been studied in detail by fitting their shape with some proper functions.

As an example, Fig. 4 shows the average LDF obtained for a sample of $\mathrm{p}$-induced shower events ('mean event') in the interval $\Delta N_{p 8}=10^{3.7}-10^{4.0}$, corresponding to a typical energy $E_{p} \sim 70 \mathrm{TeV}$. In the same plot, the LDFs for the 'mean event' induced by $\mathrm{He}$ and $\mathrm{Fe}$ primaries in the same $\Delta N_{p 8}\left(E_{H e} \sim 100 \mathrm{TeV}\right.$ and $\left.E_{F e} \sim 300 \mathrm{TeV}\right)$ are also shown.

The following function has been found to better reproduce, in the above distance interval, the LDF shape for ARGO-YBJ simulated data:

$$
\rho(r)=A \times\left(\frac{r}{r_{0}}\right)^{s^{\prime}-2}\left(1-\frac{r}{r_{0}}\right)^{s^{\prime}-4.5}
$$

with only two free parameters, normalization (A) and shape parameter $\left(s^{\prime}\right)$, while $r_{0}$ is fixed to $32 \mathrm{~m}$. It is clearly a NKG-like function, that is corresponds to the classical NKG lateral distribution function, with $r_{0} \sim 1 / 4$ the Moliere radius at the $\mathrm{YBJ}$ altitude and $s^{\prime}$ candidate to play the role of the 'lateral age' parameter.

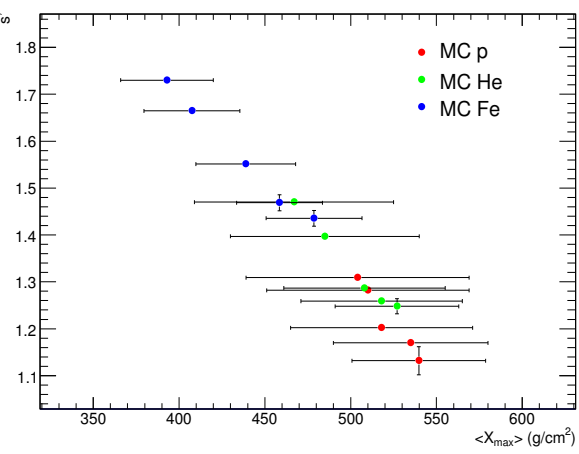

Figure 6. Age parameter $s^{\prime}$ from the fits to the lateral distributions of $\mathrm{p}, \mathrm{He}$ and $\mathrm{Fe}$ 'mean event' by the NKG-like function vs the averages of $X_{\max }$, for the five $N_{p 8}$ considered intervals.

\section{Analysis results and discussion}

The LDF of the 'mean event' for each of the three considered primaries (p, He, $\mathrm{Fe}$ ) and for each $N_{p 8}$ interval (i.e. energy bin) has been fitted by the function (1) to obtain the shape parameter $s^{\prime}$. As an example, Fig. 5 shows the average lateral distribution and the related fit for the p-primary event sample of $\Delta N_{p 8}=10^{4.7}-10^{5.0}$ corresponding to a typical energy $E_{p} \sim 500 \mathrm{TeV}$. In the same plot, the distribution and fit for the 'mean event' induced by ironnucleus primaries $\left(E_{F e} \sim 1.4 \mathrm{PeV}\right)$ can also be observed. Table 4 summarizes the results concerning the shape parameter $s^{\prime}$. The typical primary energy is also reported in the table for the different nuclei and $N_{p 8}$ intervals, as obtained from the MC simulation after the analysis cuts. The errors on the parameter $s^{\prime}$ are that arising from the described fit of the 'mean event' distribution, so they don't reflect the spread caused by the shower by shower fluctuations. Since such effects could reveal not at all negligible, they will be taken into account in a future deeper analysis. Nevertheless, from the table we deduce that, for a given primary, the $s^{\prime}$ value decreases when $N_{p 8}$ (i.e. the energy) increases, while, for a fixed $\Delta N_{p 8}$, it increases with the primary mass: this behaviour is perfectly in agreement with the expectation if $s^{\prime}$ is correlated to the shower age, thus reflecting its development stage.

This outcome has two important implications: the $s^{\prime}$ value (a) can help to constrain the shower $X_{\max }$ position and (b) can give information on the primary particle nature. Concerning the first point, we put in the same plot the $s^{\prime}$ value as obtained from the LDF fit, for each primary and $N_{p 8}$ interval, vs the average value of $X_{\max }$ distribution 


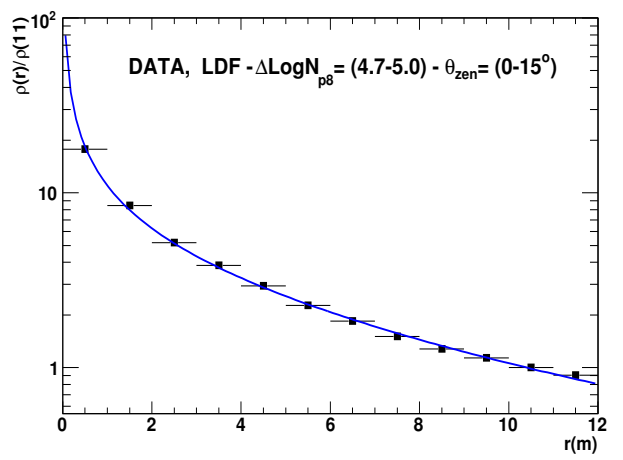

Figure 7. Reconstructed LDF of the detected particles around the core for real ARGO-YBJ events with $10^{4.7}<N_{p 8}<10^{5.0}$. The superimposed fit by the NKG-like function is also shown.

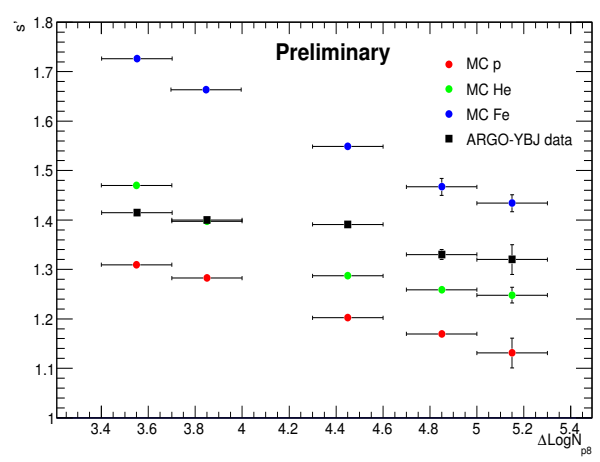

Figure 8. Age parameter $s^{\prime}$ values from the fits to the 'mean event' lateral distributions from $\mathrm{MC}$ simulations (p, He, Fe) and from experimental data, in the five $N_{p 8}$ bins.

from MC simulation, as shown in Fig. 6. The two quantities not only are clearly correlated, but also the shape parameter $s^{\prime}$ depends only on the development stage of the shower, irrespective of the nature of the primary particle and energy. In other words, that plot expresses a sort of universality of LDF of detected EAS particles in terms of the lateral shower age. This implies the possibility to select most deeply penetrating showers and quasi-constant $X_{d m}$ intervals when increasing zenith angle, an important point for correlating the exponential angular rate distribution with the interaction length of the initiating particle. To be noted that the error bars on $\left\langle X_{\max }\right\rangle$ values are the RMSs of the corresponding distributions from the MC samples, so they reflect the $X_{\text {max }}$ fluctuations which are bigger for proton than for heavier primaries, as expected.

The second implication is that $s^{\prime}$ from the LDF fit, although in the distance range up to $\sim 12 \mathrm{~m}$ of the core distance, is sensitive to the primary particle nature, thus making possible the study of primary mass composition and the selection of a proton-enriched CR primary beam.

Similar LDF distributions, in the same $N_{p 8}$ intervals used for MC data, have been obtained from real event samples and the fit with function (1) has been applied. Also for experimental data, the lateral particle density profiles appear properly described by that function, as shown in Fig. 7 where the fit to the 'mean event' in a particular $\Delta N_{p 8}$ is shown as an example. The ARGO-YBJ preliminary results in terms of the fit parameter $s^{\prime}$ for all $N_{p 8}$ intervals are summarized in the last column of Table 4. As for MC data, the error refers to the fit to the average LDF distribution for a sample of events, thus neglecting the shower by shower fluctuations.

The $s^{\prime}$ values from data are reported in Fig. 8 together with the corresponding fit results from the different primary shower events by MC simulation. Once stated the preliminary nature of this result, we observe that the data points lie between the expectations from extreme pure compositions (purely proton or iron content). In a further and more complete analysis, the systematics due to intrinsic shower fluctuations and their effects on the sensitivity to the mass composition study will be carefully evaluated.

\section{Conclusions}

The ARGO-YBJ features and setup allow the study of the shower core region with unprecedented details and the measurement with great accuracy of the radial particle density distribution. This implies the possibility to investigate several characteristics of the hadronic interactions, to extend the previous p-air cross section measurement up to $\sim \mathrm{PeV}$ proton energies and to get hints on primary mass composition.

The preliminary results from a first analog data analysis indicate the capability of ARGO-YBJ to reliably estimate the primary $\mathrm{CR}$ energy and put constraints on the detected shower $X_{\max }$ from the particle distribution structure near the core, parameterized by the lateral age $s^{\prime}$ of the LDF fit. The age parameter is also shown to be sensitive to the primary composition, even if its fluctuations, the possible dependence on the core distance and the correlations with other quantities have to be carefully studied. In the preliminary analysis presented here, the lateral age values from LDF fit to ARGO-YBJ data consistently lie between predictions from extreme pure proton or ironnucleus compositions.

\section{References}

[1] G. Aielli et al., Phys. Rev. D, 80, 092004 (2009)

[2] I. De Mitri et al., Proc. 32nd ICRC, HE 3.1 (2011)

[3] C. Bacci et al., Astropart. Phys., 17, 151 (2002)

[4] G. Aielli et al., NIM A, 562, 92 (2006)

[5] S. Mastroianni et al., Nucl. Sci. IEEE Trans. 58, 1838 (2011)

[6] M. Iacovacci et al., Proc. 32nd ICRC, HE 1.1 (2011)

[7] G. Aielli et al., NIM A, 661, S56 (2012)

[8] K. Greisen, Progress in Cosmic Ray Physics, Vol.III (NH Publishing Co, Amsterdam, 1956)

[9] R.K. Dey et al., J. Phys. G: Nucl. Part. Phys. 39, 085201 (2012)

[10] D. Heck et al., Report FZKA 6019, Forschungszentrum Karlshruhe (1998)

[11] GEANT, CERN Program Library, W5013 (1993)

[12] J.R. Hörandel, Astropart. Phys., 19, 193 (2003)

[13] S. Ostapchenko, Nucl. Phys. B, 151, 143 (2006)

[14] R. Engel et al., Proc. of 26th ICRC, Salt Lake City, vol.1, 415 (1999) 